\title{
Certain Homology Cycles of the Independence Complex of Grids
}

\author{
Jakob Jonsson
}

Received: 23 October 2008 / Revised: 29 July 2009 / Accepted: 12 August 2009 /

Published online: 3 September 2009

(C) Springer Science+Business Media, LLC 2009

\begin{abstract}
Let $G$ be an infinite graph such that the automorphism group of $G$ contains a subgroup $K \cong \mathbb{Z}^{d}$ with the property that $G / K$ is finite. We examine the homology of the independence complex $\Sigma(G / I)$ of $G / I$ for subgroups $I$ of $K$ of full rank, focusing on the case that $G$ is the square, triangular, or hexagonal grid. Specifically, we look for a certain kind of homology cycles that we refer to as "cross-cycles," the rationale for the terminology being that they are fundamental cycles of the boundary complex of some cross-polytope. For the special cases just mentioned, we determine the set $Q(G, K)$ of rational numbers $r$ such that there is a group $I$ with the property that $\Sigma(G / I)$ contains cross-cycles of degree exactly $r \cdot|G / I|-1 ;|G / I|$ denotes the size of the vertex set of $G / I$. In each of the three cases, $Q(G, K)$ turns out to be an interval of the form $[a, b] \cap \mathbb{Q}=\{r \in \mathbb{Q}: a \leq r \leq b\}$. For example, for the square grid, we obtain the interval $\left[\frac{1}{5}, \frac{1}{4}\right] \cap \mathbb{Q}$.
\end{abstract}

Keywords Grid · Independence complex · Simplicial homology · Tiling ·

Cross-polytope

\section{Introduction}

An independent set in a loopless graph $G$ is a subset of the vertex set of $G$ such that no two vertices in the subset are adjacent. The family of independent sets of a graph forms a simplicial complex, the independence complex $\Sigma(G)$ of $G$ (not to be confused with the independence complex of the cycle matroid of $G$ ).

This research was financed by a grant sponsored by Professor Günter M. Ziegler via his "Förderpreis für deutsche Wissenschaftler im Gottfried Wilhelm Leibniz-Programm der Deutschen Forschungsgemeinschaft."

J. Jonsson ( $\varangle)$

Department of Mathematics, KTH, 10044 Stockholm, Sweden

e-mail: jakobj@math.kth.se 
The purpose of this paper is to examine the homology of the independence complex of certain graphs with periodic structure. We focus on the homology generated by cycles isomorphic to fundamental cycles of the boundary complex of crosspolytopes. While such cycles do not generate all homology in general, they have a very simple structure that allows for a nice combinatorial interpretation. In particular, almost all proofs in this paper are purely combinatorial. The general problem of determining the full homology lies beyond the scope of this paper and is likely to be very hard for most of the grid graphs under consideration in this paper. See Sect. 1.5 for information about known results in certain cases.

Our motivation for studying the homology of independence complexes of grids comes from statistical physics; we refer the reader to Huijse and Schoutens [8] for more background and further references. Put briefly, the existence of homology in degree $d-1$ means that a certain supersymmetric lattice model of strongly correlated fermions exhibits ground states in which the number of particles per vertex equals $d / n$; here, $n$ is the total number of vertices in the graph under consideration.

\subsection{Grids}

First, let us describe the kind of graphs that we are interested in. Start with a simple and loopless infinite graph $G=(V, E)$, where $V$ is the set of vertices, and $E$ is the set of edges. The graph $G$ being loopless means that each edge joins two different vertices, as opposed to forming a loop at one single vertex. The graph being simple means that every pair of vertices is joined by at most one edge; there are no multiple edges. In particular, we may identify any given edge with the vertex set consisting of the two endpoints of the edge.

The degree in $G$ of a vertex $v$ is the number of edges in $E$ containing $v$. We assume that $G$ has the following properties.

- $G$ is locally finite, meaning that every vertex has finite degree.

- The automorphism group of $G$ contains a copy $K$ of $\mathbb{Z}^{d}$ as a subgroup for some $d \geq 1$, and $K$ acts freely on the vertex set of $G$.

- $G$ consists of a finite number of orbits under $K$.

For the purposes of this paper, we refer to the pair $(G, K)$ as a $d$-grid; this terminology is nonstandard. We will be mainly concerned with the case $d=2$, but we also consider the general case.

Throughout this paper, we fix a set of generators $g_{1}, \ldots, g_{d}$ of $K$; hence $K=$ $\left\langle g_{1}, \ldots, g_{d}\right\rangle$. Refer to a subgroup $I$ of $K$ of maximal $\mathbb{Z}$-rank $d$ as full-dimensional. For such a subgroup and for every vertex $a \in V$, the orbit of $a$ under $I$ is the set

$$
\operatorname{Orb}_{I}(a)=\{\kappa(a): \kappa \in I\} .
$$

The orbit of an edge $\{a, b\}$ under $I$ is the set

$$
\operatorname{Orb}_{I}(\{a, b\})=\{\{\kappa(a), \kappa(b)\}: \kappa \in I\} .
$$

Define $G / I$ to be the finite graph with vertices and edges being the orbits of $V$ and $E$, respectively, under $I$. In $G / I$, the endpoints of a given edge $\operatorname{Orb}_{I}(\{a, b\})$ are the two vertices $\operatorname{Orb}_{I}(a)$ and $\operatorname{Orb}_{I}(b)$. 
It is important to note that $G / I$ is not necessarily simple and loopless. The following lemma clarifies the situation.

Lemma 1.1 (i) The graph $G / I$ contains a loop if and only if there is an edge $\{a, b\}$ in $G$ such that $\kappa(a)=b$ for some $\kappa \in I$.

(ii) The graph $G / I$ contains multiple edges if and only if there are two distinct edges $\{a, b\}$ and $\{a, c\}$ with a common endpoint a such that $\kappa(b)=c$ for some $\kappa \in I$.

Proof (i) By definition, $G / I$ contains a loop if and only if there is an edge $\{a, b\}$ in $G$ such that $\operatorname{Orb}_{I}(a)=\operatorname{Orb}_{I}(b)$. This is equivalent to saying that there is a group element $\kappa$ such that $\kappa(a)=b$.

(ii) By definition, $G / I$ contains multiple edges if and only if there are two edges $\{a, b\}$ and $\left\{a^{\prime}, b^{\prime}\right\}$ in $G$ belonging to different orbits under $I$ such that $\operatorname{Orb}_{I}(a)=\operatorname{Orb}_{I}\left(a^{\prime}\right)$ and $\operatorname{Orb}_{I}(b)=\operatorname{Orb}_{I}\left(b^{\prime}\right)$. Let $\mu, v \in I$ be such that $\mu(a)=a^{\prime}$ and $v(b)=b^{\prime}$. Since $\operatorname{Orb}_{I}(\{a, b\}) \neq \operatorname{Orb}_{I}\left(\left\{a^{\prime}, b^{\prime}\right\}\right)$, we must have that $\mu \neq v$. Define $c=\mu^{-1}\left(b^{\prime}\right)$ and $\kappa=\mu^{-1} \circ \nu$. We have that $\left\{a^{\prime}, b^{\prime}\right\}$ belongs to the same orbit as $\left\{\mu^{-1}\left(a^{\prime}\right), \mu^{-1}\left(b^{\prime}\right)\right\}=\{a, c\}$. Since

$$
\kappa(b)=\mu^{-1} \circ v(b)=\mu^{-1}\left(b^{\prime}\right)=c,
$$

the claim follows.

We will restrict our attention to groups $I$ such that $G / I$ is indeed simple and loopless. In this situation, also in $G / I$ we may identify each edge with its set of endpoints.

\subsection{Cross-Cycles}

The ideal goal for any grid $G$ would be to determine the homology of $\Sigma(G / I)$ for every $I$. Unfortunately, this appears to be a very difficult problem in general. For this reason, we confine ourselves to a far less ambitious project. Specifically, we focus on a certain kind of homology cycles that we refer to as "cross-cycles." This terminology is chosen to align with the fact that each such cycle coincides with the fundamental cycle of the boundary complex of a cross-polytope.

Specifically, a cross-cycle of size $k$ in a simplicial complex $\Delta$ is an element $z$ in the simplicial chain group $\tilde{C}_{k-1}(\Delta ; \mathbb{Z})$ such that the following hold.

- We have that

$$
z=\left(\left[a_{1}\right]-\left[b_{1}\right]\right) \wedge\left(\left[a_{2}\right]-\left[b_{2}\right]\right) \wedge \cdots \wedge\left(\left[a_{k}\right]-\left[b_{k}\right]\right)
$$

for distinct elements $a_{1}, \ldots, a_{k}, b_{1}, \ldots, b_{k}$ from the vertex set of $\Delta$.

- Some set of the form $\left\{a_{i}: i \in X\right\} \cup\left\{b_{j}: j \in\{1, \ldots, k\} \backslash X\right\}$ forms a maximal face of $\Delta$.

Here, $\left[x_{1}\right] \wedge\left[x_{2}\right] \wedge \cdots \wedge\left[x_{k}\right]$ denotes the oriented simplex $\left[x_{1}, \ldots, x_{k}\right]$, which is a generator of $\tilde{C}_{k-1}(\Delta ; \mathbb{Z})$ if and only if $\left\{x_{1}, \ldots, x_{k}\right\}$ is a member of $\Delta$. In particular, 
$\left\{a_{i}: i \in X\right\} \cup\left\{b_{i}: i \in\{1, \ldots, k\} \backslash X\right\}$ belongs to $\Delta$ for every $X \subseteq\{1, \ldots, k\}$. See Munkres [13, Sect. 5] for more information on simplicial homology.

After appropriate relabeling, one may replace the second condition with the condition that the set $\left\{a_{1}, a_{2}, \ldots, a_{k}\right\}$ be a maximal face of $\Delta$. Since $z$ is a cycle and no nonzero multiple of $z$ is a boundary in $\tilde{C}_{k-1}(\Delta ; \mathbb{Z})$, we obtain that $z$ generates an infinite cyclic subgroup of $\tilde{H}_{k-1}(\Delta ; \mathbb{Z})$. In particular, $\tilde{H}_{k-1}(\Delta ; \mathbb{Z})$ has nonzero rank.

For a general complex $\Delta$, all homology is not necessarily generated by crosscycles. In particular, a complete description of all cross-cycles in $\Delta$ is not necessarily sufficient to completely describe the homology of $\Delta$ or even the set of degrees with nonvanishing homology. Nevertheless, in the case of independence complexes of graphs, cross-cycles admit a nice combinatorial interpretation that makes them particularly interesting; see Proposition 3.1.

\subsection{Possible Ratios of Elements in Cross-Cycles}

Let $(G, K)$ be a $d$-grid. For a full-dimensional subgroup $I$ of $K$, define $|G / I|$ to be the number of vertices in $G / I$. Our goal is to examine possible sizes of cross-cycles in $\Sigma(G / I)$, expressed in terms of the size of the vertex set of $G / I$. More precisely, we define $Q(G, K)$ to be the set of rational numbers $r$ with the following property:

- There exists a full-dimensional subgroup $I$ of $K$ such that $G / I$ is simple and loopless and such that $\Sigma(G / I)$ contains a cross-cycle of size exactly $r \cdot|G / I|$.

Note that $r$ is the ratio of elements from the vertex set of $G / I$ in any set appearing in the cross-cycle. As we will see, if there exists one subgroup $I$ with the above property, then there are infinitely many such subgroups; see Sect. 3.2. In particular, there are no "pathological" values in $Q(G, K)$.

\subsection{Main Results}

As already mentioned, we focus on 2-grids. Specifically, we consider the square grid Sq, the triangular grid Tri, and the hexagonal grid Hex; see Fig. 1 for illustrations. This selection of grids is quite natural, the three grids being the most important and well-studied 2-grids in the literature. In each case, there is a natural action of $K=\mathbb{Z}^{2}$ on the grid as illustrated in Fig. 2.

For real numbers $a \leq b$, write $[a, b]=\{r: a \leq r \leq b\}$. The following theorem is the main result of this paper.

Theorem 1.2 The following hold.

- $Q(\mathrm{Sq}, K)=\left[\frac{1}{5}, \frac{1}{4}\right] \cap \mathbb{Q}$.

- $Q($ Tri, $K)=\left[\frac{1}{7}, \frac{1}{5}\right] \cap \mathbb{Q}$.

- $Q(\operatorname{Hex}, K)=\left[\frac{1}{4}, \frac{5}{18}\right] \cap \mathbb{Q}$.

For example, for every rational number between $1 / 5$ and $1 / 4$, there is a group $I$ such that there are cross-cycles of size $r \cdot|\mathrm{Sq} / I|$ in $\Sigma(\mathrm{Sq} / I)$. Note that $|\mathrm{Sq} / I|$ and $|\operatorname{Tri} / I|$ both equal $\left|\mathbb{Z}^{2} / I\right|$, whereas $|\mathrm{Hex} / I|$ equals $2 \cdot\left|\mathbb{Z}^{2} / I\right|$; see Fig. 2 . We prove the different parts of Theorem 1.2 in Sects. 5, 6, and 7. 

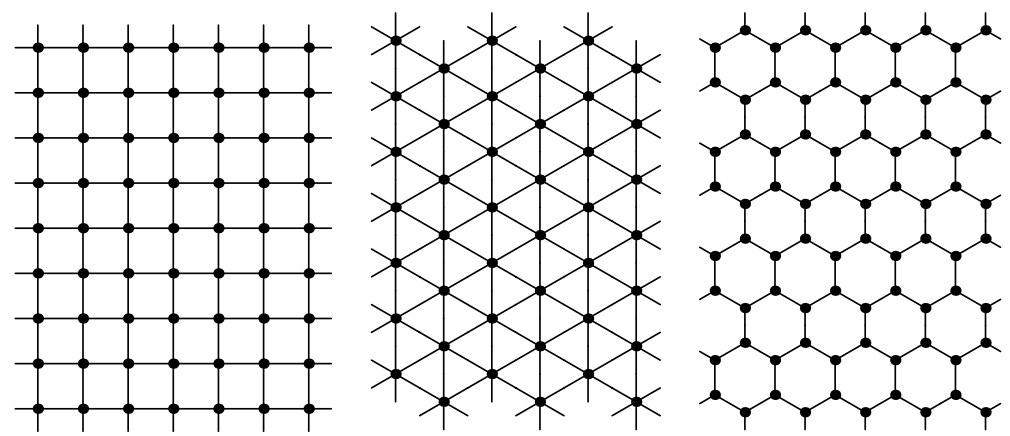

Fig. 1 From left to right the square grid Sq, the triangular grid Tri, and the hexagonal grid Hex

Fig. 2 The free group with generators $g_{1}$ and $g_{2}$ acting on Sq, Tri, and Hex
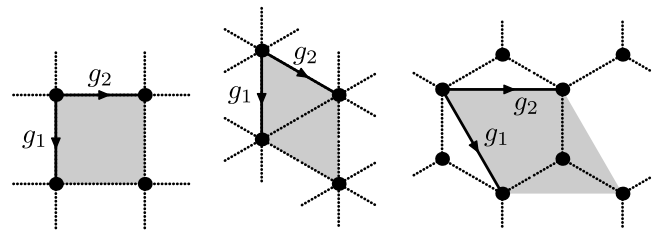

\subsection{Related Work}

Independence complexes of graphs are important objects, mainly because they coincide with flag complexes, which are simplicial complexes in which any given set $\sigma$ is a face if and only if every subset of $\sigma$ of size two is a face. For example, the barycentric subdivision of any simplicial complex is a flag complex. For a random selection of results on flag complexes from the viewpoint of independence in graphs, see Ehrenborg and Hetyei [2], Engström [3], Kozlov [12], and Živaljevič [16].

The homology of independence complexes of grids tends to be hard to compute in general, but there are exceptions. Huijse, Halverson, Fendley, and Schoutens [9, 10] analyzed the homology of the independence complex of square grids and obtained a concrete formula in the case that the subgroup $I$ is generated by two vectors $(-m, m)$ and $(a, b)$ such that $m \geq 1$ and $a+b$ is a nonzero multiple of 3 . The authors related the homology to certain rhombus tilings [11] that we will discuss in some detail in Sect. 5. Their method relies on the assumption that one of the generators of $I$ is of the form $( \pm m, \pm m)$; as of today, it remains unclear whether it is possible to extend their method to arbitrary groups $I$. Bousquet-Mélou, Linusson, and Nevo [1] examined the homology of other square grids with free and cylindrical boundary conditions. Fendley and Schoutens [5] computed the (co-)homology of the independence complex of octagon-square and enneagon-triangle grids; see Sect. 8 for discussion. For numerical estimates on the homology for various grids, see Engström [4] and van Eerten [15].

Fundamental cycles of cross-polytopes are important in the work of Shareshian and Wachs [14] on the matching and chessboard complexes, which are independence complexes on the line graph of complete and bipartite graphs, respectively. 


\section{Basic Properties of Full-Dimensional Subgroups}

Throughout this section, $(G, K)$ is a $d$-grid. The proof of the following lemma is completely straightforward.

Lemma 2.1 For every finite set $\left\{I_{1}, \ldots, I_{r}\right\}$ of full-dimensional subgroups of $K$, the intersection $\bigcap_{j} I_{j}$ is a full-dimensional subgroup of $K$.

Let $\operatorname{Orb}_{I}(a)$ denote the orbit containing the vertex $a$. Given a subgroup $I$ of $K$, we have that $G / I$ is loopless if and only if $\operatorname{Orb}_{I}(a)$ is an independent set in $G$ for each vertex $a$. Moreover, $G / I$ is simple if and only if there is at most one edge between $a$ and $\operatorname{Orb}_{I}(b)$ for every $a, b \in V$.

Lemma 2.2 There exists a full-dimensional subgroup I of $K$ such that $G / I$ is simple and loopless.

Proof By Lemma 1.1, it suffices to show that there is a full-dimensional subgroup $I$ of $K$ such that the following hold for each vertex $a \in V$ :

(i) For every vertex $b$ adjacent to $a$, we have that $b \notin \operatorname{Orb}_{I}(a)$.

(ii) For every two distinct vertices $b$ and $c$, both adjacent to $a$, we have that $c \notin$ $\mathrm{Orb}_{I}(b)$.

Let $A$ be a subset of $V$ consisting of one vertex from each orbit in $G$ under $K$. Note that properties (i) and (ii) hold for each vertex $a \in V$ if and only if they hold for each vertex $a \in A$. By assumption, $A$ is finite, and each vertex $a \in A$ has finitely many neighbors. In particular, there are finitely many pairs $(a, b)$ to consider in (i) and finitely many pairs $(b, c)$ to consider in (ii). Since $K$ acts freely on $G$, there are finitely many group elements $\kappa \in K$, all nonzero, such that $\kappa(a)=b$ for some pair $(a, b)$ as in (i) or $\kappa(b)=c$ for some pair $(b, c)$ as in (ii). Let $S$ be the set of such group elements $\kappa$. Since $S$ is finite and does not contain the zero element, we immediately deduce that there is a number $s>0$ such that $S \cap(s \cdot K)=\emptyset$. In particular, (i) and (ii) hold for $I=s \cdot K$.

Lemma 2.3 Let I be a full-dimensional subgroup of $K$. If $G / I$ is simple and loopless, then $G / J$ is simple and loopless for every full-dimensional subgroup $J$ of $I$.

Proof Suppose that $a$ and $b$ are adjacent elements such that $b \in \operatorname{Orb}_{J}(a)$. Since $I$ contains $J$, this implies that $b \in \operatorname{Orb}_{I}(a)$. Analogously, if $a$ has at least two neighbors in $\operatorname{Orb}_{J}(a)$, then the same is true in $\operatorname{Orb}_{I}(a)$.

\section{Basic Properties of Cross-Cycles}

We examine the structure of cross-cycles, proving different simple results about their behavior. First, we present a characterization of cross-cycles. 
Proposition 3.1 Let $G$ be a finite simple and loopless graph, and let $A:=$ $\left\{a_{1}, \ldots, a_{k}\right\}$ and $B:=\left\{b_{1}, \ldots, b_{k}\right\}$ be disjoint vertex sets in $G$ of size $k$. Then $z:=\bigwedge_{i=1}^{k}\left(\left[a_{i}\right]-\left[b_{i}\right]\right)$ is a cross-cycle in $\Sigma(G)$ if and only if the following hold.

- The edges of $G(A \cup B)$ are $\left\{a_{1}, b_{1}\right\}, \ldots,\left\{a_{k}, b_{k}\right\}$.

- Some set of the form $\left\{a_{i}: i \in X\right\} \cup\left\{b_{j}: j \in\{1, \ldots, k\} \backslash X\right\}$ is maximal with respect to set inclusion among independent sets in $G$ (i.e., the set forms a maximal face of $\Sigma(G))$.

Proof Suppose that $z$ is a cross-cycle in $\Sigma(G)$. Since

$$
z= \pm \partial\left(\left[a_{j}\right] \wedge\left[b_{j}\right] \wedge \bigwedge_{i \neq j}\left(\left[a_{i}\right]-\left[b_{i}\right]\right)\right)
$$

we have that $a_{j}$ and $b_{j}$ are adjacent for every $j \in\{1, \ldots, k\}$. Moreover, expanding $z$, we note that every set of the form $\left\{a_{i}: i \in X\right\} \cup\left\{b_{j}: j \in\{1, \ldots, k\} \backslash X\right\}$ is an independent set. By definition, one of these sets is a maximal independent set. Conversely, one easily checks that $z$ is a cross-cycle if the given conditions hold.

\subsection{Bounds on the Size of Cross-Cycles}

There are a multitude of ways to provide bounds on the size of cross-cycles. We present some quite elementary bounds that will turn out to be useful in later sections.

Throughout this section, $G=(V, E)$ is a finite simple and loopless graph. For a vertex $x$ in $G$, the star Star $_{x}$ with center $x$ is the vertex set consisting of $x$ along with all its neighbors, i.e.,

$$
\operatorname{Star}_{x}:=\{x\} \cup\{y:\{x, y\} \in E\} .
$$

Proposition 3.2 Suppose that $X$ is a set of vertices in $G$ such that $\operatorname{Star}_{x} \cap \operatorname{Star}_{y}=\varnothing$ whenever $x, y \in X$ and $x \neq y$. Then all cross-cycles in $\Sigma(G)$ have size at least $|X|$.

Proof Let $z:=\bigwedge_{i \in Y}\left(\left[a_{i}\right]-\left[b_{i}\right]\right)$ be a cross-cycle in $\Sigma(G)$. By Proposition 3.1, we may assume that $A:=\left\{a_{i}: i \in Y\right\}$ is a maximal independent set in $G$. As a consequence, $A \cap \operatorname{Star}_{x}$ is nonempty for every $x \in X$, because otherwise $A \cup\{x\}$ would be an independent set properly containing $A$, contradicting the maximality of $A$. This immediately yields that $|A| \geq|X|$.

Proposition 3.3 Suppose that the degree of each vertex in $G$ is at least $\delta$ and at most $\epsilon$. Then every cross-cycle has size at most $|G| \cdot \frac{\epsilon}{2(\epsilon+\delta-1)}$. In particular, if $\delta=\epsilon$, then every cross-cycle has size at most $|G| \cdot \frac{\delta}{4 \delta-2}$.

Proof Suppose that there is a cross-cycle of size $k$. Let $A$ and $B$ be sets as in Proposition 3.1, and let $C:=V \backslash(A \cup B)$. By Proposition 3.1, there are at least $2 k(\delta-1)$ edges from $A \cup B$ to $C$. It follows that $C$ consists of at least $2 k(\delta-1) / \epsilon$ vertices. As a consequence,

$$
|G|=|A \cup B|+|C|=2 k+|C| \geq 2 k+\frac{2 k(\delta-1)}{\epsilon}=k \cdot \frac{2(\epsilon+\delta-1)}{\epsilon} .
$$


Proposition 3.4 Suppose that $V$ can be written as a disjoint union of $\operatorname{sets}\left\{C_{i}: i \in Y\right\}$ such that each $C_{i}$ has the following property for some integer $k_{i}$.

- The induced subgraph $G(D)$ has a vertex of degree at least two whenever $D$ is a subset of $C_{i}$ of size at least $k_{i}+1$.

Then all cross-cycles in $\Sigma(G)$ have size at most $\frac{1}{2} \sum_{i \in Y} k_{i}$.

Proof Let $z$ be a cross-cycle, and let $X$ be the set of vertices appearing in the cycle. By Proposition 3.1, $G(X)$ is a perfect matching, meaning that every vertex has degree one. As a consequence, $X \cap C_{i}$ consists of at most $k_{i}$ elements for each $i \in Y$.

\subsection{Cross-Cycles in Grids}

Let $(G, K)$ be a $d$-grid. We show that there are infinitely many groups $J$ such that $G / J$ contains a cross-cycle of size $r \cdot|G / J|$ whenever $r \in Q(G, K)$.

Proposition 3.5 Let I be a full-dimensional subgroup of $K$ such that $G / I$ is simple and loopless. Let $r$ be a rational number. Suppose that $\Sigma(G / I)$ contains a cross-cycle of size $r \cdot|G / I|$. Then $\Sigma(G / J)$ contains a cross-cycle of size $r \cdot|G / J|$ whenever $J$ is a full-dimensional subgroup of $I$.

Proof Let $A:=\left\{a_{1}, \ldots, a_{k}\right\}$ and $B:=\left\{b_{1}, \ldots, b_{k}\right\}$ be vertices in $G / I$ such that $z:=$ $\bigwedge_{i=1}^{k}\left(\left[a_{i}\right]-\left[b_{i}\right]\right)$ is a cross-cycle in $\Sigma(G / I)$ and such that $k=r \cdot|G / J|$. We may assume that $A$ is a maximal independent set in $G / I$. Let $A^{\prime}$ and $B^{\prime}$ be the union of all orbits under $I$ containing some element from $A$ and $B$, respectively. Since $G / I$ is simple and loopless, $G\left(A^{\prime} \cup B^{\prime}\right)$ is a perfect matching, and each edge is of the form $\{a, b\}$ with $a \in A^{\prime}$ and $b \in B^{\prime}$. Moreover, $A^{\prime}$ is a maximal independent set in $G$, because every $c$ in $V(G)$ is adjacent to some element in $\operatorname{Orb}_{I}(a)$. In particular, whenever $G / J$ is simple and loopless, the sets $\left\{\operatorname{Orb}_{J}(a): a \in A^{\prime}\right\}$ and $\left\{\operatorname{Orb}_{J}(b)\right.$ : $\left.b \in B^{\prime}\right\}$ satisfy Proposition 3.1. Applying Lemma 2.3, we are done.

The assumption about $G / I$ being simple is essential. Namely, let $G$ be the graph with vertex set $\{0,1\} \times \mathbb{Z}$ and with an edge between $(s, i)$ and $(t, j)$ whenever $|j-i| \leq 1$ and $s \neq t$. With the natural action on $G$ by $\mathbb{Z}$, we have that $G / \mathbb{Z}$ is a graph with two vertices joined by three edges. In particular, $\Sigma(G / \mathbb{Z})$ contains a cross-cycle of size $1=\frac{1}{2} \cdot|G / \mathbb{Z}|$. However, for $k \geq 2, \Sigma(G / k \mathbb{Z})$ does not contain any cross-cycles of size $\frac{1}{2} \cdot|G / k \mathbb{Z}|$, because $G / k \mathbb{Z}$ is not a perfect matching; apply Proposition 3.3.

Corollary 3.6 Let I be a full-dimensional subgroup of $K$ such that $G / I$ is simple and loopless. If every full-dimensional subgroup $J$ of I has the property that $\Sigma(G / J)$ contains no cross-cycle of size $r \cdot|G / J|$, then $r \notin Q(G, K)$.

Proof Suppose that $G / I^{\prime}$ contains a cross-cycle of size $r \cdot\left|G / I^{\prime}\right|$ for some fulldimensional group $I^{\prime}$ such that $G / I^{\prime}$ is simple and loopless. By Proposition 3.5, $G / J^{\prime}$ contains a cross-cycle of size $r \cdot\left|G / J^{\prime}\right|$ whenever $J^{\prime}$ is a full-dimensional subgroup of $I^{\prime}$. In particular, this is true for the intersection $J$ of $I$ and $I^{\prime}$, which is a 
full-dimensional subgroup of $K$ by Lemma 2.1. However, $J$ is also a subgroup of $I$, which is a contradiction to the assumption about $I$.

\subsection{Disjoint Unions of Graphs and Similar Constructions}

Finally, we show how properties of cross-cycles are preserved when "joining" two graphs.

Lemma 3.7 Let $G=(V, E)$ be a finite graph such that $V$ is the disjoint union of the two sets $U$ and $W$ with the property that there are no edges between $U$ and $W$. Then $z$ is a cross-cycle in $\Sigma(G)$ if and only if $z=z_{1} \wedge z_{2}$, where $z_{i}$ is a cross-cycle in $\Sigma\left(G_{i}\right)$ for $i \in\{1,2\}$. In particular, there are cross-cycles of size $k$ in $\Sigma(G)$ if and only if there are integers $k_{1}$ and $k_{2}$ summing to $k$ such that there are cross-cycles of size $k_{i}$ in $\Sigma\left(G_{i}\right)$ for $i \in\{1,2\}$.

Proof Suppose that $z_{1}$ and $z_{2}$ are cross-cycles in $\Sigma\left(G_{1}\right)$ and $\Sigma\left(G_{2}\right)$, respectively. Since there are no edges between $G_{1}$ and $G_{2}$, one easily checks that $z_{1} \wedge z_{2}$ satisfies the conditions in Proposition 3.1. Conversely, given a cross-cycle $z:=\bigwedge_{i}\left(\left[a_{i}\right]-\right.$ $\left[b_{i}\right]$ ), Proposition 3.1 yields that each pair $\left\{a_{i}, b_{i}\right\}$ must form an edge either in $G_{1}$ or in $G_{2}$. Hence we may write $z=z_{1} \wedge z_{2}$, where $z_{i}$ is a cycle in $\Sigma\left(G_{i}\right)$ for each $i \in\{1,2\}$. Again, it is straightforward to check that $z_{1}$ and $z_{2}$ satisfy the conditions in Proposition 3.1.

Lemma 3.8 Let $G=(V, E)$ be a finite graph and assume that $V$ is the disjoint union of two nonempty sets $U$ and $W$ with the property that every $u$ in $U$ and $w$ in $W$ are joined by an edge. Then $z$ is a cross-cycle in $\Sigma(G)$ if and only if either of the following holds.

- $z$ is a cross-cycle in either $\Sigma(G(U))$ or $\Sigma(G(W))$.

- $z=[a]-[b]$, where $a \in U$ and $b \in W$, and either all vertices in $U \backslash\{a\}$ are adjacent to a or all vertices in $W \backslash\{b\}$ are adjacent to $b$.

Proof This is again a straightforward consequence of Proposition 3.1.

\section{General Properties of $Q(G, K)$}

We consider the following general question.

For which subsets $R$ of $\mathbb{Q}$ is there a grid $(G, K)$ such that $Q(G, K)=R$ ?

In this section, we provide a partial answer to this question, proving that such a graph exists whenever $R$ is a closed interval $[a, b] \cap \mathbb{Q}$ such that $0<a \leq b<\frac{1}{2}$ and also when $R=\left\{\frac{1}{2}\right\}$. We also provide an example demonstrating that $Q(G, K)$ is not always of the form $[a, b] \cap \mathbb{Q}$.

Theorem 4.1 For every d-grid $(G, K)$, we have that $Q(G, K) \subseteq\left(0, \frac{1}{2}\right)$ unless $G$ is a perfect matching, in which case $Q(G, K)=\left\{\frac{1}{2}\right\}$. Moreover, for every two rational 
numbers $a$ and $b$ such that $0<a \leq b<\frac{1}{2}$, there is a graph $G$ such that $Q(G, K)=$ $[a, b] \cap \mathbb{Q}$.

Proof Throughout this proof, $I$ is a group such that $G / I$ is simple and loopless; such an $I$ exists by Lemma 2.2. First, note that $\Sigma(G / I)$ contains more than just the empty set. In particular, $0 \notin Q(G, K)$. Also, note that if $G$ contains an isolated vertex, then $\Sigma(G / I)$ is a cone and has no nonvanishing homology. As a consequence, there are no cross-cycles in $\Sigma(G / I)$. From now on, we always assume that all vertices in $G$ have degree at least one.

By Proposition 3.3, $\Sigma(G / I)$ has a cross-cycle of size $\frac{|G / I|}{2}$ only if $G / I$ is a perfect matching. Now, $G / I$ is a perfect matching if and only if $G$ itself is a perfect matching, because the homomorphism $G \rightarrow G / I$ is degree-preserving. Clearly, $\Sigma(G / I)$ is then the boundary of a cross-polytope of dimension $\frac{|G / I|}{2}$. As a consequence, $Q(G, K)=$ $\frac{1}{2}$ in this case. Another application of Proposition 3.3 hence yields the first statement in the theorem.

For the second statement, let $a$ and $b$ be rational numbers such that $0 \leq a \leq b<\frac{1}{2}$; we can write $a=p_{1} / q$ and $b=p_{2} / q$ for integers $p_{1}, p_{2}, q$ such that $2 \leq p_{1} \leq p_{2} \leq$ $q / 2$. Define a graph $G_{1}$ as the disjoint union of the following three pieces.

- The first piece $H_{1}$ consists of $p_{1}-2$ copies of $K_{2}$, the complete graph on two vertices. The number of vertices in $H_{1}$ is $2 p_{1}-4$.

- The second piece $H_{2}$ consists of $p_{2}-p_{1}+1$ copies of $K_{2}$ and an additional vertex adjacent to all other vertices in $H_{2}$. The number of vertices in $H_{2}$ is $2 p_{2}-2 p_{1}+3$.

- The third piece $H_{3}$ is a complete graph on $q-2 p_{2}+1$ vertices.

Applying Lemma 3.7, we obtain that the one cross-cycle (up to sign) in $\Sigma\left(H_{1}\right)$ has size $p_{1}-2$. By the same lemma, all cross-cycles in $\Sigma\left(H_{3}\right)$ have the same size one. Applying Lemmas 3.7 and 3.8, we obtain that $\Sigma\left(H_{2}\right)$ has cross-cycles of size one and $p_{2}-p_{1}+1$ but of no other sizes. By Lemma 3.7, we may conclude that $\Sigma\left(G_{1}\right)$ contains cross-cycles of size

$$
\left(p_{1}-2\right)+1+1=p_{1}
$$

and

$$
\left(p_{1}-2\right)+\left(p_{2}-p_{1}+1\right)+1=p_{2} .
$$

Moreover, the number of vertices in $G_{1}$ is

$$
\left(2 p_{1}-4\right)+\left(2 p_{2}-2 p_{1}+3\right)+\left(q-2 p_{2}+1\right)=q .
$$

Now, let $G$ be the infinite graph with one disjoint copy $G_{1}^{(i)}$ of $G_{1}$ for each $i \in \mathbb{Z}$. Let $K \cong \mathbb{Z}$ be the group generated by the automorphism $\tau$ acting on $G$ by mapping $G_{1}^{(i)}$ to $G_{1}^{(i+1)}$ in the natural manner for all $i \in \mathbb{Z}$.

Let $r$ be a rational number such that $a \leq r \leq b$; we can write $r=\frac{k a+(m-k) b}{m}$ for some integers $k$ and $m$ satisfying $0 \leq k \leq m$. In fact, there are infinitely many ways to express $r$ in this manner: replace $k$ and $m$ with $T k$ and $T m$ for any integer $T$. It is clear that $G_{m}:=G /\left\langle\tau^{m}\right\rangle$ consists of $m$ disjoint copies of $G_{1}$. Applying Lemma 3.7 
Fig. 3 The graph $G$ in Proposition 4.2. One "period" of $G$ is marked in grey

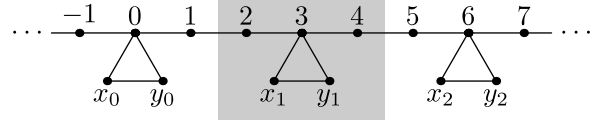

(or Proposition 3.5 applied to $G_{1}$ and $\left\langle\tau^{m}\right\rangle$ ), we obtain that $\Sigma\left(G_{m}\right)$ contains crosscycles of size $k p_{1}+(m-k) p_{2}$. Since $G_{m}$ contains $m q$ vertices, we obtain the fraction

$$
\frac{k p_{1}+(m-k) p_{2}}{m q}=\frac{k a+(m-k) b}{m}=r ;
$$

hence $r \in Q(G, K)$. Conversely, another application of Lemma 3.7 yields that there are no cross-cycles of $\Sigma\left(G_{m}\right)$ of size below $m p_{1}$ or above $m p_{2}$; hence $r \notin Q(G, K)$ unless $a \leq r \leq b$. As a consequence, $Q(G, K)=[a, b] \cap \mathbb{Q}$.

We proceed with an example showing that $Q(G, K)$ is not necessarily of the form $[a, b] \cap \mathbb{Q}$. Let $G$ be the infinite graph constructed in the following manner. Start with the infinite path $P_{\infty}$; this graph has vertex set $\mathbb{Z}$ and edge set $\{\{i, i+1\}: i \in \mathbb{Z}\}$. For each $k \in \mathbb{Z}$, add two vertices $x_{k}$ and $y_{k}$ and the three edges $\left\{3 k, x_{k}\right\},\left\{3 k, y_{k}\right\}$, and $\left\{x_{k}, y_{k}\right\}$. The resulting graph is $G$. Consider the group $K \cong \mathbb{Z}$ generated by the automorphism $\tau$ acting on $G$ by mapping $i$ to $i+3$ for all $i \in \mathbb{Z}$ and by mapping $x_{k}$ and $y_{k}$ to $x_{k+1}$ and $y_{k+1}$, respectively, for all $k$. See Fig. 3 for an illustration.

Proposition 4.2 With $G$ defined as above, we have that $Q(G, K)=\left\{\frac{1}{5}, \frac{2}{5}\right\}$.

Proof Let $m \geq 1$. Write $G_{m}:=G /\left\langle\tau^{m}\right\rangle$. Let $z$ be a cross-cycle of size $k$ in $\Sigma\left(G_{m}\right)$, and let $\left\{a_{i}, b_{i}\right\}$ be the corresponding pairs; $1 \leq i \leq k$. By Proposition 3.1, we may assume that $A:=\left\{a_{1}, \ldots, a_{k}\right\}$ is a maximal independent set in $G_{m}$. Write $B:=\left\{b_{1}, \ldots, b_{k}\right\}$.

Suppose that there is a $j$ such that $3 j-3 \in A$, whereas $3 j \notin A$. If $3 j \in B$, then $3 j-1 \in A$ by the maximality of $A$. Moreover, for the same reason, either $x_{j}$ or $y_{j}$ belongs to $A$. Yet, this is a contradiction, because $3 j$ would then have two neighbors in $A$. Hence $3 j \notin B$. Since $3 j-2$ is adjacent to $3 j-3$, which belongs to $A$, we cannot have that $3 j-1 \in A$. However, this means that the two neighbors of $3 j-1$ are outside $A$, which contradicts the maximality of $A$.

As a consequence, either $A$ contains $3 \mathbb{Z}_{m}$ or $A$ has empty intersection with $3 \mathbb{Z}_{m}$. In the former case, $A$ must be equal to $3 \mathbb{Z}_{m}$ and hence have size $m$, the set $3 \mathbb{Z}_{m}$ being a maximal independent set in $G_{m}$. In the latter case, the graph obtained by removing the set $3 \mathbb{Z}_{m}$ is a perfect matching on $4 m$ elements. As a consequence, $A$ must have size $2 m$. Since $|G / I|=5 m$, we conclude that $Q(G, K) \subseteq\left\{\frac{1}{5}, \frac{2}{5}\right\}$. Conversely, the two pairings $\left\{\{3 j, 3 j+1\}: j \in \mathbb{Z}_{m}\right\}$ and $\left\{\left\{x_{j}, y_{j}\right\},\{3 j+1,3 j+2\}: j \in \mathbb{Z}_{m}\right\}$ are easily seen to satisfy Proposition 3.1, which yields that $Q(G, K) \supseteq\left\{\frac{1}{5}, \frac{2}{5}\right\}$.

The above construction can be refined in several ways. For example, replacing the piece on the vertex set $\left\{x_{k}, y_{k}\right\}$ with a perfect matching $H_{k}$ of size $n$ and adding edges from each vertex in $H_{k}$ to the vertex $3 k$, we obtain a graph $G$ with the property that $Q(G, K)=\left\{\frac{1}{2 n+3}, \frac{n+1}{2 n+3}\right\}$. Replacing $H_{k}$ with an arbitrary graph may yield more 
complicated patterns such as a set $Q(G, K)$ of the form $\{q\} \cup([a, b] \cap \mathbb{Q})$. Instead of going into detail on this matter, let us state a question about the general structure of $Q(G, K)$.

Does every grid $(G, K)$ have the property that $Q(G, K)$ equals $\bigcup_{i=1}^{k}\left[a_{i}, b_{i}\right] \cap \mathbb{Q}$ for some rational numbers $a_{1}, b_{1}, \ldots, a_{k}, b_{k}$ such that $a_{i} \leq b_{i}$ for $1 \leq i \leq k$ ? In particular, is $Q(G, K)$ always a closed set?

\section{The Square Grid}

Recall that Sq is the infinite square 2-grid illustrated on the left in Fig. 1 with a group $K \cong \mathbb{Z}^{2}$ acting on Sq as illustrated on the left in Fig. 2 . We identify $K$ with $\mathbb{Z}^{2}$ and let $(a, b)$ denote the group element $g_{1}^{a} g_{2}^{b}$. Let $u:=\left(u_{1}, u_{2}\right)$ and $v:=\left(v_{1}, v_{2}\right)$ be two linearly independent vectors in $\mathbb{Z}^{2}$. Note that $|\mathrm{Sq} /\langle u, v\rangle|$ equals $\left|u_{1} v_{2}-u_{2} v_{1}\right|$. Write $\Sigma_{u, v}:=\Sigma(\mathrm{Sq} /\langle u, v\rangle)$.

To avoid misconceptions, we state already at this point that we label elements in $\mathbb{Z}^{2}$ according to the matrix convention; this should be clear from the way we defined $g_{1}$ and $g_{2}$ in Fig. 2. We label the vertices of Sq in the same manner.

For the special case $u=(m, 0)$ and $v=(0, n)$, Fendley, Schoutens, and van Eerten [6] studied properties of $\Sigma_{m, n}:=\Sigma_{u, v}$ in the context of the "hard-square model," a well-studied model of statistical mechanics. They stated a conjecture about the Euler characteristic of $\Sigma_{m, n}$, which the author of the present paper settled in a previous paper [11]. Specifically, the Euler characteristic of the more general complex $\Sigma_{u, v}$ was related to certain rhombus tilings of the plane. We will not go into detail on this result, but we will need to make use of the very same rhombus tilings, which are defined in the following manner.

- The entire plane is tiled.

- The corners of each rhombus all belong to $\mathbb{Z}^{2}$.

- Each rhombus has side length $\sqrt{5}$, meaning that each side is parallel to and has the same length as either $(1,2),(-1,2),(2,1)$, or $(-2,1)$.

- The intersection of two rhombi is either empty, a common corner, or a common side.

One easily checks that a rhombus tiling is uniquely determined by the set of rhombus corners in the tiling. From now on, we always identify a tiling with this set.

We refer to a rhombus of area $k$ as a $k$-rhombus. Up to translation, there are six kinds of rhombi in which all corners are integer points and all sides have length $\sqrt{5}$ : two 3-rhombi, two 4-rhombi, and two 5-rhombi; see Fig. 4. Note that the 5-rhombi are squares. We restrict our attention to tilings of the plane with 4- and 5-rhombi (i.e., the four rightmost rhombi in Fig. 4); see Fig. 5 for an example. We refer to tilings with 4- and 5-rhombi as balanced. We say that a rhombus tiling is $(u, v)$-invariant if the tiling is invariant under the two translations $x \mapsto x+u$ and $x \mapsto x+v$.

As alluded to above, the Euler characteristic of $\Sigma_{u, v}$ can be expressed in terms of balanced $(u, v)$-invariant rhombus tilings [11]. The proof of this correspondence relied on a matching on $\Sigma_{u, v}$ with the property that all balanced $(u, v)$-invariant rhombus tilings modulo $\langle u, v\rangle$ remained unmatched. Readers familiar with discrete Morse 
Fig. 4 Six different rhombi

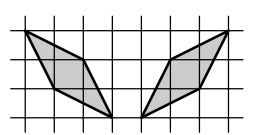

3-rhombi

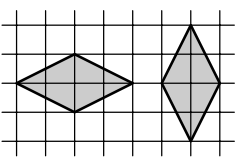

4-rhombi

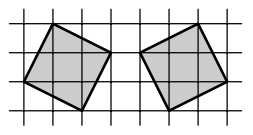

5-rhombi

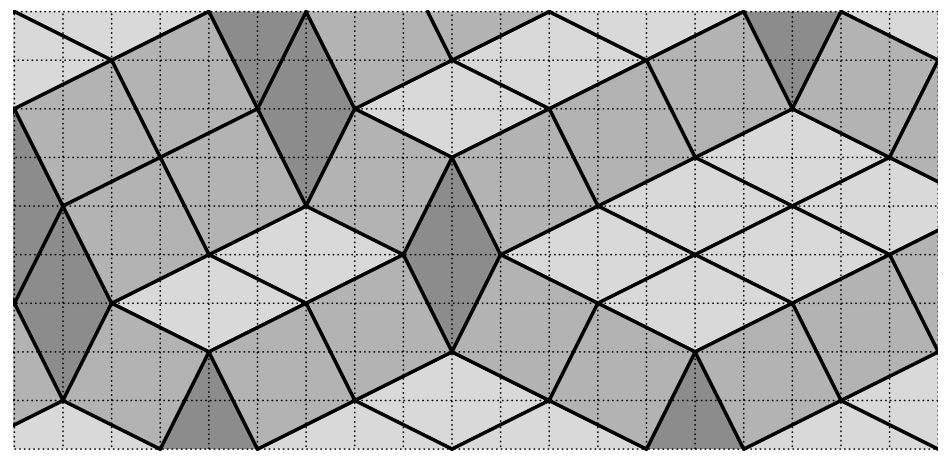

Fig. 5 Portion of a balanced rhombus tiling

theory [7] may ask whether this matching reveals any information about the topology of $\Sigma_{u, v}$. The answer is that the matching does not correspond to a discrete Morse function. In particular, we cannot use discrete Morse theory to deduce that the rhombus tilings correspond to nonvanishing cycles in the homology of $\Sigma_{u, v}$.

Yet, as we will see in this section, each tiling does correspond to such a nonvanishing cycle, in fact a cross-cycle, though there might be linear dependencies between some of the cycles. As already mentioned, Huijse et al. [8, 10] have managed to express the full homology in terms of rhombus tilings for certain choices of $u$ and $v$.

\subsection{Lower Bound on $Q(\mathrm{Sq}, K)$}

We demonstrate that $Q(\mathrm{Sq}, K)$ contains every rational number $r$ between $\frac{1}{5}$ and $\frac{1}{4}$. For any element $x \in \mathbb{Z}^{2}$, define $\mathrm{e}(x):=x+(0,1)$ (east), $\mathrm{n}(x):=x+(-1,0)$ (north), $\mathrm{w}(x):=x+(0,-1)$ (west), and $\mathrm{s}(x):=x+(1,0)$ (south); recall our matrix convention for rows and columns.

Proposition 5.1 For each $k \geq 1$, if there is a balanced $(u, v)$-invariant rhombus tiling $\rho$ with exactly $k$ congruence classes modulo $\langle u, v\rangle$, then there exists a cross-cycle of size $k$.

Proof Let $\rho$ be a balanced $(u, v)$-invariant rhombus tiling with $k$ congruence classes modulo $\langle u, v\rangle$; we identify $\rho$ with this set of congruence classes. Let $\operatorname{se}(\rho)$ be the set obtained by applying se $=\mathrm{s}$ o e to each element in $\rho$. For an element $x \in \rho$, let $\hat{x}$ be the unique element in $\rho \cap\left\{\mathrm{s}^{2} \mathrm{e}(x), \mathrm{se}^{2}(x)\right\}$. It is clear that $\hat{x}$ is the only neighbor 


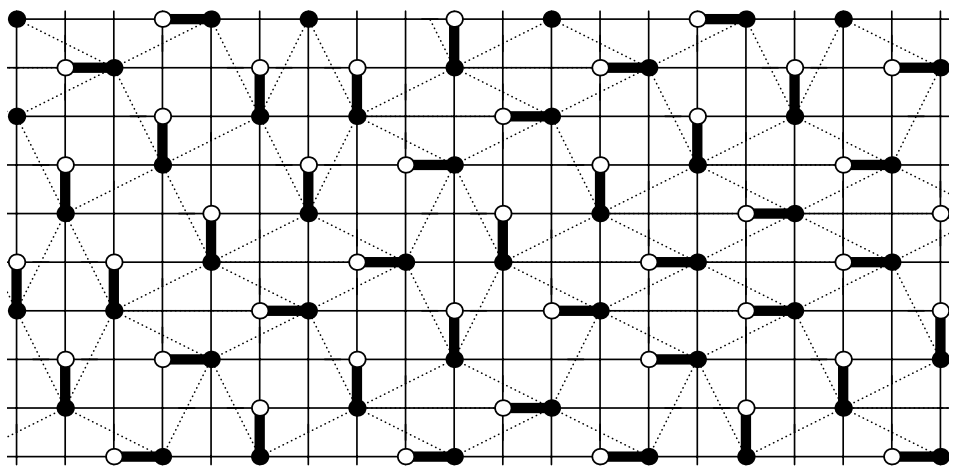

Fig. 6 The partition of $\rho \cup \operatorname{se}(\rho)$ into edges $\{\hat{x}$, se $(x)\}$ as described in the proof of Proposition $5.1 ; \rho$ is the rhombus tiling in Fig. 5. Black points represent elements of $\rho$, whereas white points represent elements of $\operatorname{se}(\rho)$

of $\operatorname{se}(x)$ in $\rho$. As a consequence, the partition

$$
\rho \cup \operatorname{se}(\rho)=\bigcup_{x \in \rho}\{\hat{x}, \operatorname{se}(x)\}
$$

into edges has the property that there are no edges between elements from different edges; see Fig. 6 for an illustration. In particular, since $\rho$ and $\operatorname{se}(\rho)$ are maximal in $\Sigma_{u, v}$, Proposition 3.1 applies.

Remark Using the same method as in the above proof, one obtains the same property for the cycle

$$
z_{\rho}^{\mathrm{W}}:=\bigwedge_{x \in \rho}([\tilde{x}]-[\operatorname{sw}(x)])
$$

where $\tilde{x}$ is the unique element in $\rho \cap\left\{\mathrm{s}^{2} \mathrm{w}(x), \mathrm{sw}^{2}(x)\right\}$. Let $R_{u, v}^{k}$ be the family of balanced $(u, v)$-invariant rhombus tilings with exactly $k$ congruence classes modulo $\langle u, v\rangle$. An interesting problem is to examine the rank of the subgroup of $\tilde{H}_{k-1}\left(\Sigma_{u, v} ; \mathbb{Z}\right)$ generated by $\left\{z_{\rho}^{\mathrm{e}}, z_{\rho}^{\mathrm{w}}: \rho \in R_{u, v}^{k}\right\}$ and relate this rank to the size of $R_{u, v}^{k}$.

Proposition 5.2 (Jonsson [11, Theorem 2.6]) Let $a, b, c, d$ be arbitrary nonnegative integers such that $a+b \geq 1$ and $c+d \geq 1$. Write $x:=a(1,2)+b(2,1)$ and $y:=$ $c(-1,2)+d(-2,1)$. If $\langle u, v\rangle$ is a subgroup of $\langle x, y\rangle$, then there are $(u, v)$-invariant rhombus tilings such that the number of 4-rhombi and 5-rhombi modulo $\langle u, v\rangle$ equals $a c+b d$ and $a d+b c$, respectively.

Corollary 5.3 With assumptions and notation as in Proposition 5.2, there are crosscycles of size $r \cdot|\mathrm{Sq} /\langle u, v\rangle|$ in $\Sigma_{u, v}$, where $\frac{1}{r}=\frac{a d+b c}{(a+b)(c+d)}+4$. In particular, $Q(\mathrm{Sq}, K) \supseteq\left[\frac{1}{5}, \frac{1}{4}\right] \cap \mathbb{Q}$. 


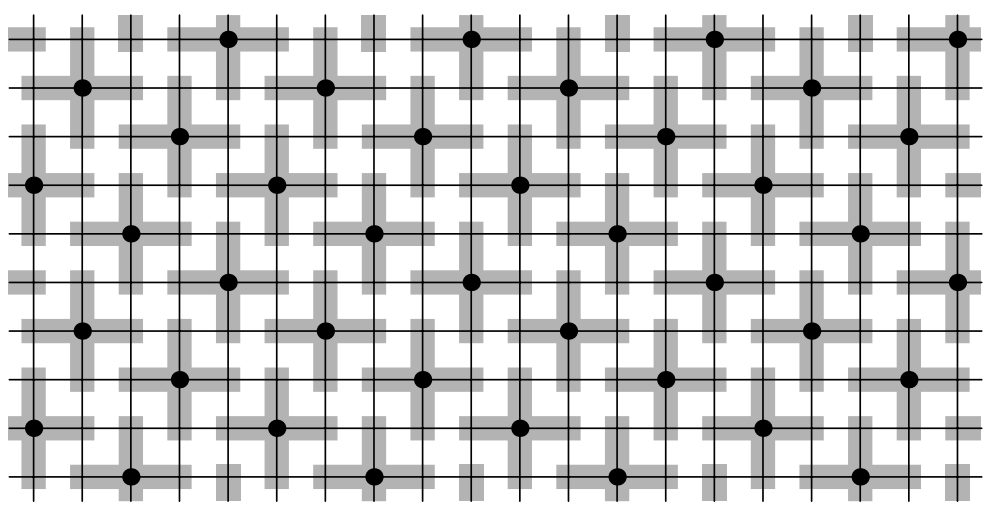

Fig. 7 Partition of Sq into stars. The black points form the set $X$ in the proof of Lemma 5.4

Proof This is an immediate consequence of Propositions 5.1 and 5.2. For the last statement, let $p$ and $q$ be positive integers such that $\frac{1}{5} \leq \frac{p}{q} \leq \frac{1}{4}$. Choosing $a=1$, $b=0, c=5 p-q$, and $d=q-4 p$, we obtain that $r=\frac{p}{q}$.

\subsection{Upper Bound on $Q(\mathrm{Sq}, K)$}

It remains to prove that $Q(\mathrm{Sq}, K) \subseteq\left[\frac{1}{5}, \frac{1}{4}\right] \cup \mathbb{Q}$.

\section{Lemma 5.4 The following hold.}

- If $\langle u, v\rangle$ is contained in $\langle(1,2),(-2,1)\rangle$ or $\langle(2,1),(-1,2)\rangle$, then all cross-cycles in $\mathrm{Sq} /\langle u, v\rangle$ have size at least $\frac{1}{5} \cdot|\mathrm{Sq} /\langle u, v\rangle|$.

- If $\langle u, v\rangle$ is contained in $\langle(1,2),(-1,2)\rangle$ or $\langle(2,1),(-2,1)\rangle$, then all cross-cycles in $\mathrm{Sq} /\langle u, v\rangle$ have size at most $\frac{1}{4} \cdot|\mathrm{Sq} /\langle u, v\rangle|$.

As a consequence, $Q(\mathrm{Sq}, K) \subseteq\left[\frac{1}{5}, \frac{1}{4}\right] \cap \mathbb{Q}$.

Proof For the first statement, we want to apply Proposition 3.2. Using symmetry, we may assume that $\langle u, v\rangle$ is contained in $\langle(1,2),(-2,1)\rangle$.

Note that the star $\operatorname{Star}_{x}$ contains $x$ and the four neighbors $\mathrm{e}(x), \mathrm{n}(x), \mathrm{w}(x)$, and $\mathrm{s}(x)$. Define $X$ to be the subset of $V_{u, v}$ consisting of all $(a, b)$ such that $a+2 b \equiv 0 \quad(\bmod 5)$. This is well defined, because $(1,2)$ and $(-2,1)$ satisfy this condition. By Proposition 3.2, we need only prove that Star $x$ and $\operatorname{Star}_{x^{\prime}}$ are disjoint whenever $x$ and $x^{\prime}$ are distinct elements in $X$. Clearly, $x$ and $x^{\prime}$ are not neighbors. Suppose that $x$ and $x^{\prime}$ have a common neighbor $t$. This would mean that $x-x^{\prime}$ is plus or minus one of the vectors $(-1,1),(2,0),(1,1)$, and $(0,2)$. As a consequence, $\left|(1,2) \cdot\left(x_{i}-x_{j}\right)\right| \in\{1,2,3,4\}$. This contradicts the fact that $(1,2) \cdot\left(x-x^{\prime}\right)$ is divisible by five, which concludes the proof of the first statement. See Fig. 7 for an illustration.

For the second statement, we want to apply Proposition 3.4. Using symmetry, we may assume that $\langle u, v\rangle$ is contained in $\langle(1,2),(-1,2)\rangle$. 


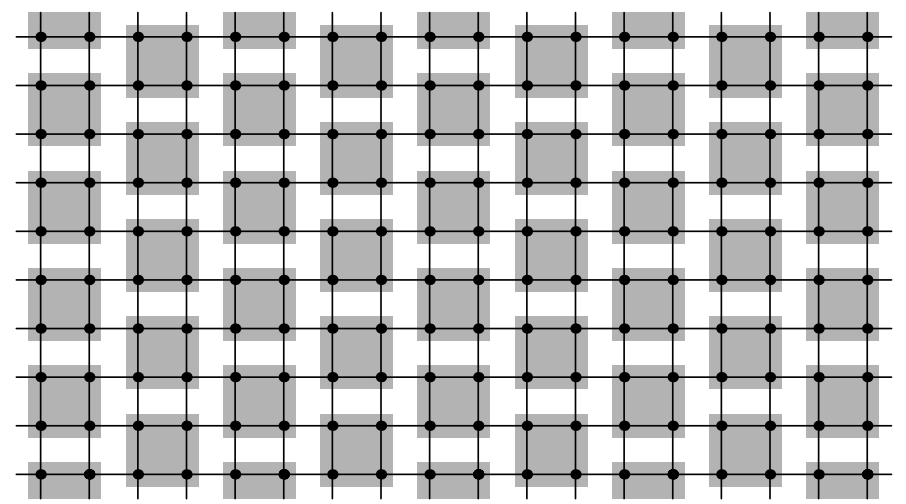

Fig. 8 Partition of Sq into sets $C_{r, s}$

Table 1 The polynomial $\sum_{k \geq 0} \operatorname{rank} \tilde{H}_{k}\left(\Sigma_{m, n} ; \mathbb{Z}\right) t^{k}$ for small $m$ and $n$. In all known cases, the homology is torsion-free

\begin{tabular}{|c|c|c|c|c|c|c|c|c|c|}
\hline & $n=2$ & 3 & 4 & 5 & 6 & 7 & 8 & 9 & 10 \\
\hline$m=2$ & 1 & $t$ & $3 t$ & $t$ & $t^{2}$ & $t^{3}$ & $3 t^{3}$ & $t^{3}$ & $t^{4}$ \\
\hline 3 & $t$ & $4 t$ & $t$ & $t^{3}$ & $4 t^{3}$ & $t^{3}$ & $t^{5}$ & $4 t^{5}$ & $t^{5}$ \\
\hline 4 & $3 t$ & $t$ & $7 t^{3}$ & $2 t^{3}+t^{4}$ & $t^{4}+4 t^{5}$ & $2 t^{5}+t^{6}$ & $t^{6}+8 t^{7}$ & & \\
\hline 5 & $t$ & $t^{3}$ & $2 t^{3}+t^{4}$ & $9 t^{4}$ & $t^{5}$ & & & & \\
\hline
\end{tabular}

For each point $(r, s) \in V_{u, v}$ such that $2 r+s$ is divisible by four, form the set $C_{r, s}:=\{(r, s),(r, s+1),(r+1, s),(r+1, s+1)\}$. This means that we partition the whole of $\mathrm{Sq} /\langle u, v\rangle$ into squares as illustrated in Fig. 8. Since $2 r+s$ is divisible by four when $(r, s)=(1,2)$ or $(-1,2)$, this is well defined. One easily checks that $C_{r, s}$ satisfies Proposition 3.4 with $k_{r, s}=2 ; \mathrm{Sq}\left(C_{r, s}\right)$ is a square graph. The second statement follows.

For the final statement, apply Corollary 3.6.

Combining Corollary 5.3 and Lemma 5.4, we obtain the main result of this section.

Theorem 5.5 We have that $Q(\mathrm{Sq}, K)=\left[\frac{1}{5}, \frac{1}{4}\right] \cap \mathbb{Q}$.

\subsection{More on the Homology of $\Sigma_{u, v}$}

While Huijse et al. [8, 10] found a formula for the homology of $\Sigma_{u, v}$ for large classes of groups $\langle u, v\rangle$, the rectangular case $u=(m, 0)$ and $v=(0, n)$ remains unsolved. Using computer, we have calculated the homology of $\Sigma_{m, n}:=\Sigma_{u, v}$ for small values of $m$ and $n$; see Table 1. Note that the reduced Euler characteristic of $\Sigma_{m, n}$ is -1 whenever $m$ and $n$ are coprime. By results from a previous paper [11], this is always true and is a consequence of the following fact. 
Proposition 5.6 (Jonsson [11, Theorem 2.7]) Whenever $m$ and $n$ are coprime, there are no balanced $((m, 0),(0, n))$-invariant rhombus tilings.

By Proposition 5.6, Proposition 5.1 does not tell us anything about the homology of $\Sigma_{m, n}$ when $m$ and $n$ are coprime. Given the simplicity of the Euler characteristic, one may ask whether the homology is equally simple. Clearly, as Table 1 illustrates, the homology is not always that of a sphere in odd dimension; for $(m, n) \in\{(4,5),(4,7)\}$, there is homology in two different degrees. The possibility remains that the homology is always concentrated in just a few degrees for $m$ and $n$ coprime.

\section{The Triangular Grid}

This section is devoted to the triangular grid Tri.

Lemma 6.1 We have that $Q($ Tri, $K) \supseteq\left[\frac{1}{7}, \frac{1}{5}\right] \cap \mathbb{Q}$.

Proof Recall the definition of the generators $g_{1}$ and $g_{2}$ of $K$ from Fig. $2 ; g_{1}$ means moving one step down, whereas $g_{2}$ means moving one step in the south-east direction. Identify one point as the origin $(0,0)$, and define $(i, j):=g_{1}^{i} \circ g_{2}^{j}(0,0)$.

Let $\alpha$ and $\beta$ be nonnegative integers such that $\alpha+\beta \geq 2$. Write $q:=5 \alpha+7 \beta$, and define $u:=(2,1)$ and $v:=(q, 0)$. It is clear that Tri $\langle u, v\rangle$ contains $q$ vertices. Moreover, the set of neighbors of a given vertex $x$ is

$$
\begin{aligned}
x & +\{(-1,0),(1,0),(0,1),(0,-1),(-1,1),(1,-1)\} \\
& =x+\{(-1,0),(1,0),(-2,0),(2,0),(-3,0),(3,0)\} .
\end{aligned}
$$

As a consequence, since $q \geq 10$, Tri $\langle\langle u, v\rangle$ is simple and loopless. Note that we may identify Tri $\langle u, v\rangle$ with the graph $C_{q, 3}$ with vertex set $\mathbb{Z}_{q}$ and with an edge between $i$ and $j$ if and only if $i-j \in\{ \pm 1, \pm 2, \pm 3\}$ (modulo $q$ ). The independence complex of $C_{q, 3}$ appears in Kozlov's work [12, Question 3] as $\mathcal{C}_{q}^{4}$.

Define

$$
A:=\{5 i: 0 \leq i \leq \alpha-1\} \cup\{5 \alpha+7 j: 0 \leq j \leq \beta-1\}
$$

and

$$
B:=A+1=\{5 i+1: 0 \leq i \leq \alpha-1\} \cup\{5 \alpha+7 j+1: 0 \leq j \leq \beta-1\}
$$

these are subsets of the vertex set $\mathbb{Z}_{q}$ of $C_{q, 3}$. It is clear that $A$ is a maximal independent set in $C_{q, 3}$ (as is $B$ ) and that $C_{q, 3}(A \cup B$ ) is a perfect matching. Hence the conditions in Proposition 3.1 are satisfied. It follows that $C_{q, 3}$ contains a cross-cycle of size $|A|=\alpha+\beta$, which yields that

$$
\frac{\alpha+\beta}{5 \alpha+7 \beta} \in Q(\text { Tri, } K) \text {. }
$$




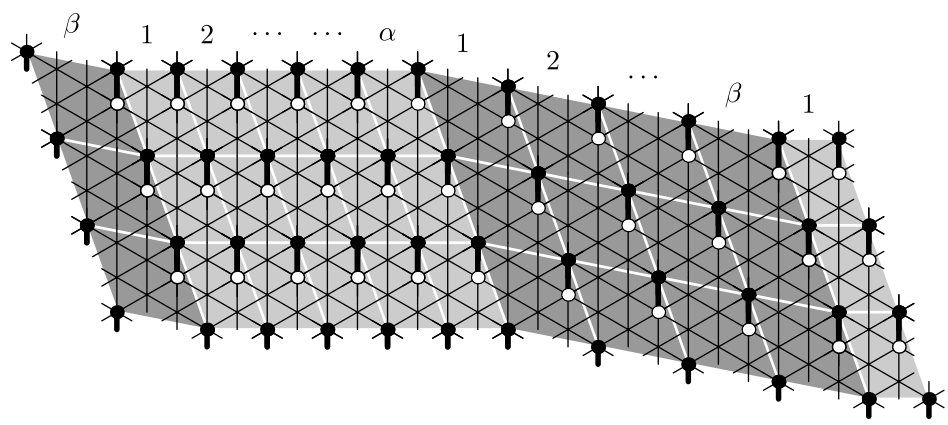

Fig. 9 The set $A \cup B$ in the proof of Lemma 6.1; black points represent elements in $A$, whereas white points represent elements in $B$. Note the induced tiling of the plane into parallelograms

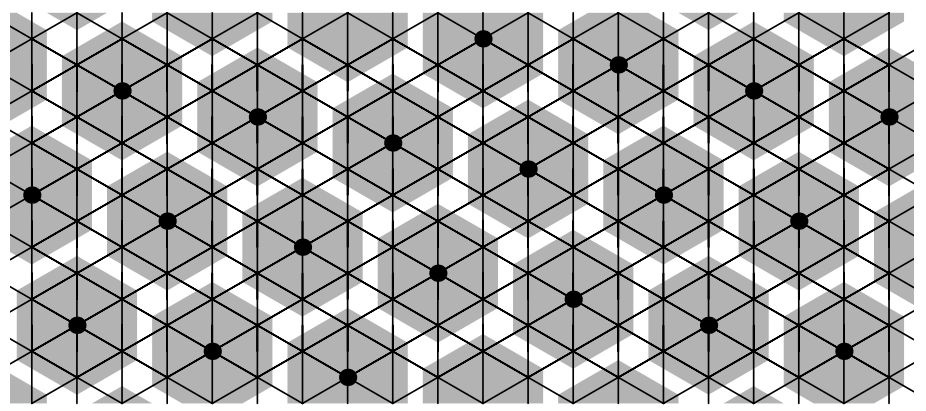

Fig. 10 Partition of Tri into stars. The black points form the set $Z$ in the proof of Lemma 6.2

In particular, for any positive integers $p$ and $q$ such that $\frac{1}{7} \leq \frac{p}{q} \leq \frac{1}{5}$, the choice $\alpha=$ $7 p-q$ and $\beta=q-5 p$ yields that $\frac{p}{q} \in Q($ Tri, $K)$.

It might be worth noting that the set $A \cup B$ in the proof of Lemma 6.1, lifted to Tri, has the shape illustrated in Fig. 9.

Lemma 6.2 We have that $Q(\operatorname{Tri}, K) \subseteq\left[\frac{1}{7}, \frac{1}{5}\right] \cap \mathbb{Q}$.

Proof Suppose that $r \in Q(\operatorname{Tri}, K)$. To show that $r \geq \frac{1}{7}$, consider the partition of Tri into stars as illustrated in Fig. 10; let $Z$ be the set of centers. This partition is clearly periodic, and any group $I$ such that $Z$ is invariant under $I$ has the property that Tri/ $I$ has no cross-cycles of size below $\mid$ Tri $/ I \mid / 7$; apply Proposition 3.2 and use the fact that every seventh element of Tri belongs to $Z$. Applying Corollary 3.6, we obtain that $r$ must be at least $\frac{1}{7}$.

To show that $r \leq \frac{1}{5}$, suppose that we are given a cross-cycle $z$ of size $k$ in Tri/I. Let $X$ be the set of vertices that are present in $z$, and let $Y$ be the remaining set of vertices; we have that $|X|=2 k$ and $|Y|=|\operatorname{Tri} / I|-2 k$. It is clear that an element $y$ in $Y$ cannot be adjacent to more than four elements in $X$. Namely, the neighborhood of $y$ is a six-gon, meaning that every induced subgraph on five vertices in this neighbor- 
Fig. 11 The situation around the vertices $y$ and $y_{1}$ in the proof of Lemma 6.2. The black points represent elements in $X$, the white points represent elements in $Y$, and the grey points represent points with an unknown status

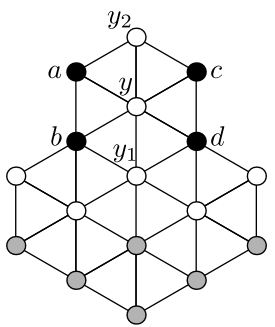

hood contains a vertex of degree two. We want to show that at most one third of the elements in $Y$ are adjacent to four elements in $X$. Since each element in $X$ has five neighbors in $Y$, this will yield that

$$
\begin{aligned}
5 \cdot 2 k \leq\left(3 \cdot \frac{2}{3}+4 \cdot \frac{1}{3}\right) \cdot|Y| \\
\quad \Longleftrightarrow \quad 10 k \leq \frac{10}{3} \cdot(|\operatorname{Tri} / I|-2 k) \\
\Longleftrightarrow \quad k \leq \frac{1}{5} \cdot|\operatorname{Tri} / I| .
\end{aligned}
$$

Here, the left-hand side of the first two rows counts the number of edges $x y$ such that $x \in X$ and $y \in Y$.

To obtain the desired result, let $Y_{4}$ be the set of vertices in $Y$ with four neighbors in $X$. Let $y$ be an element in $Y_{4}$. One easily checks that the only possibility is that $X \cap$ Star $_{y}$ is a union of two edges $\{a, b\}$ and $\{c, d\}$; up to rotation, the situation is as in Fig. 11. Let $y_{1}$ and $y_{2}$ be the two neighbors of $y$ in $Y$. Inspecting Fig. 11, one readily verifies that $y_{1} \notin Y_{4}$ and that the same is true for all neighbors of $y_{1}$ except $y$. Namely, there is no room for two disjoint edges from $X$ in the neighborhood of any of these neighbors. By symmetry, the same is true for $y_{2}$. Since each element in $Y_{4}$ has two neighbors in $Y \backslash Y_{4}$ and since no element in $Y \backslash Y_{4}$ has more than one neighbor in $Y_{4}$, it follows that $2\left|Y_{4}\right| \leq\left|Y \backslash Y_{4}\right|$. Here, the left-hand side counts the number of edges $y y^{\prime}$ such that $y \in Y_{4}$ and $y^{\prime} \in Y \backslash Y_{4}$. This concludes the proof.

Combining Lemmas 6.1 and 6.2, we obtain the following result.

Theorem 6.3 We have that $Q(\operatorname{Tri}, K)=\left[\frac{1}{7}, \frac{1}{5}\right] \cap \mathbb{Q}$.

\section{The Hexagonal Grid}

We proceed with the hexagonal grid Hex.

Lemma 7.1 We have that $Q(\mathrm{Hex}, K) \supseteq\left[\frac{1}{4}, \frac{5}{18}\right] \cap \mathbb{Q}$.

Proof Let $\alpha, \beta$ be nonnegative integers, not both zero, and consider the matching on Hex illustrated in Fig. 13; the building blocks of this matching are given in Fig. 12. We 
Fig. 12 Building blocks of the matching on Hex in Fig. 13. Each block consists of 72 vertices. In block $X$, there are 20 matched pairs, whereas block Y contains 18 matched pairs

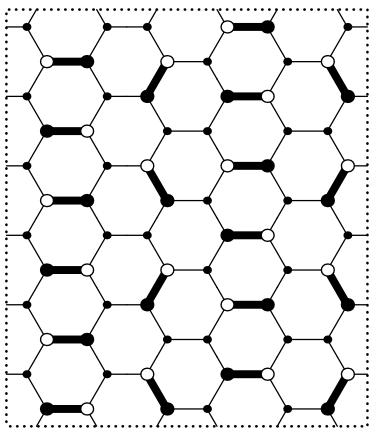

Block X

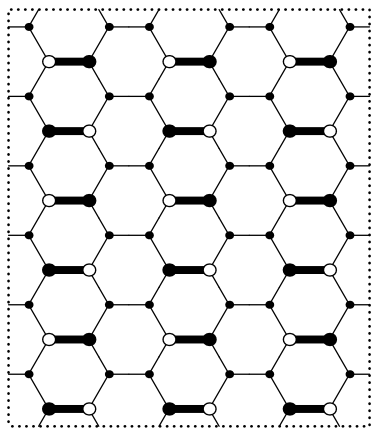

Block Y

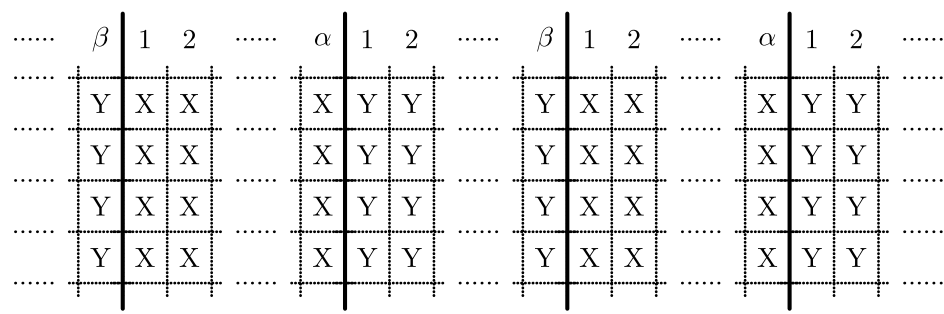

Fig. 13 Periodic matching on Hex using the building blocks in Fig. 12. This matching satisfies Proposition 3.1

thus have a repeating pattern consisting of $\alpha$ columns of " $\mathrm{X}$ " blocks followed by $\beta$ columns of "Y" blocks. One easily checks that this matching satisfies Proposition 3.1; black points represent elements in $A$, and white points represent elements in $B$.

Now, each " $\mathrm{X}$ " block contains 20 matched pairs, each "Y" block contains 18 matched pairs, and the number of vertices in each block is 72 . As a consequence, if $I$ is a full-dimensional group that leaves the given matching invariant, then the number of pairs in the induced matching on $\mathrm{Hex} / I$ equals $|\mathrm{Hex} / I|$ times

$$
\frac{20 \alpha+18 \beta}{72 \alpha+72 \beta}=\frac{1}{36} \cdot \frac{10 \alpha+9 \beta}{\alpha+\beta} .
$$

In particular, for any positive integers $p$ and $q$ such that $\frac{1}{4}=\frac{9}{36} \leq \frac{p}{q} \leq \frac{10}{36}=\frac{5}{18}$, the choice $\alpha=36 p-9 q$ and $\beta=10 q-36 p$ yields that the number of pairs equals $\frac{p}{q} \cdot|\mathrm{Hex} / I|$. By Proposition 3.1, we are done.

Lemma 7.2 We have that $Q(\mathrm{Hex}, K) \subseteq\left[\frac{1}{4}, \frac{5}{18}\right] \cap \mathbb{Q}$.

Proof Suppose that $r \in Q(\mathrm{Hex}, K)$. To show that $r \geq \frac{1}{4}$, consider the partition of Hex into stars as illustrated in Fig. 14; let $Z$ be the set of centers. Proceeding as in the proof of Lemma 6.2, we deduce that $r$ is at least $\frac{1}{4}$.

To show that $r \leq \frac{5}{18}$, suppose that we are given a cross-cycle $z$ of size $k$ in Hex/I. Let $X$ be the set of vertices that are present in $z$, and let $Y$ be the remaining set of 
Fig. 14 Partition of Hex into stars. The large points form the set $Z$ in the proof of Lemma 7.2
Fig. 15 On the left, a hexagon from the set $\mathcal{H}$ in the proof of Lemma 7.2. On the right, the situation around the vertices $y_{1}$ and $y_{2}$. The color conventions for vertices are the same as in Fig. 11; black vertices belong to $X$
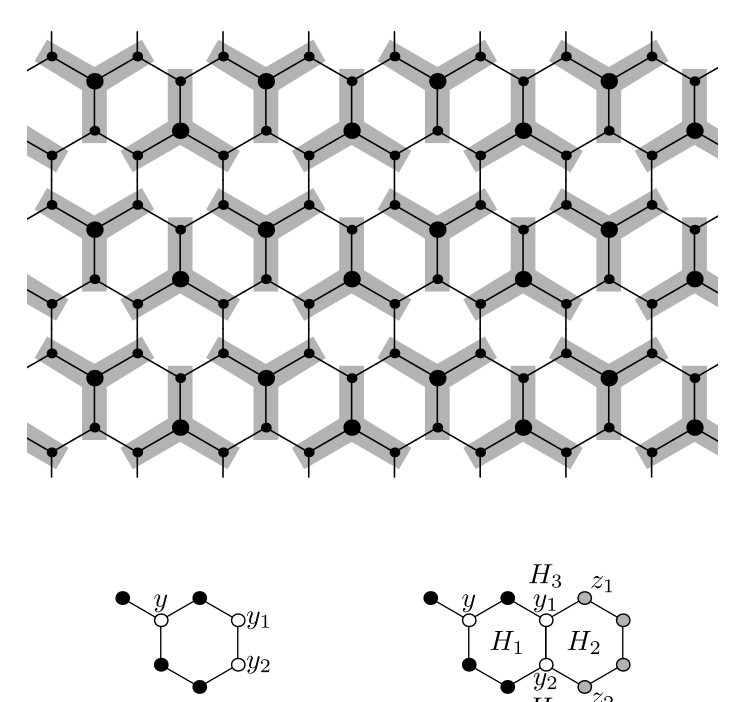

vertices; we have that $|X|=2 k$ and $|Y|=|\mathrm{Hex} / I|-2 k$. We want to show that at most half of the elements in $Y$ have the property that all three neighbors belong to $X$. Since each element in $X$ has two neighbors in $Y$, this will yield that

$$
\begin{aligned}
2 \cdot 2 k & \leq\left(2 \cdot \frac{1}{2}+3 \cdot \frac{1}{2}\right) \cdot|Y| \\
& \Longleftrightarrow \quad 4 k \leq \frac{5}{2} \cdot(|\mathrm{Hex} / I|-2 k) \\
& \Longleftrightarrow \quad k \leq \frac{5}{18} \cdot|\mathrm{Hex} / I| .
\end{aligned}
$$

Let $Y_{3}$ be the set of vertices in $Y$ with all three neighbors in $X$. Let $\mathcal{H}$ be the set of hexagons in Hex/I with the property that exactly one of the six vertices of the hexagon, $y$, belongs to $Y_{3}$ and the other two elements, $y_{1}$ and $y_{2}$, belong to $Y \backslash Y_{3}$ and are adjacent to each other. Up to rotation and reflection, the situation is as in the picture on the left in Fig. 15.

First, we show that every vertex in $Y \backslash Y_{3}$ belongs to at most two hexagons in $\mathcal{H}$. Consider a hexagon $H_{1}$ such as the one on the left in Fig. 15 and look at the hexagon $H_{2}$ just to the right of $H_{1}$ as illustrated in the picture on the right in Fig. 15. With notation as in that picture, note that $z_{1}$ and $z_{2}$ must both be vertices in $X$ for $H_{2}$ to belong $\mathcal{H}$. However, if this is the case, then the hexagons $H_{3}$ and $H_{4}$ do not belong to $\mathcal{H}$, because $y_{1}$ and $y_{2}$ do not belong to $Y_{3}$. The desired claim follows.

Next, we show that every vertex in $Y_{3}$ belongs to at least one hexagon in $\mathcal{H}$. Up to rotation and reflection, the situation is as in the picture on the left in Fig. 16. If $z_{1} \notin X$, then $H_{2}$ belongs to $\mathcal{H}$. Hence assume that $z_{1} \in X$. This yields the situation in 
Fig. 16 On the left, the situation around a vertex $y$ in $Y_{3}$. On the right, the same situation but with the additional assumption that $z_{1} \in X$
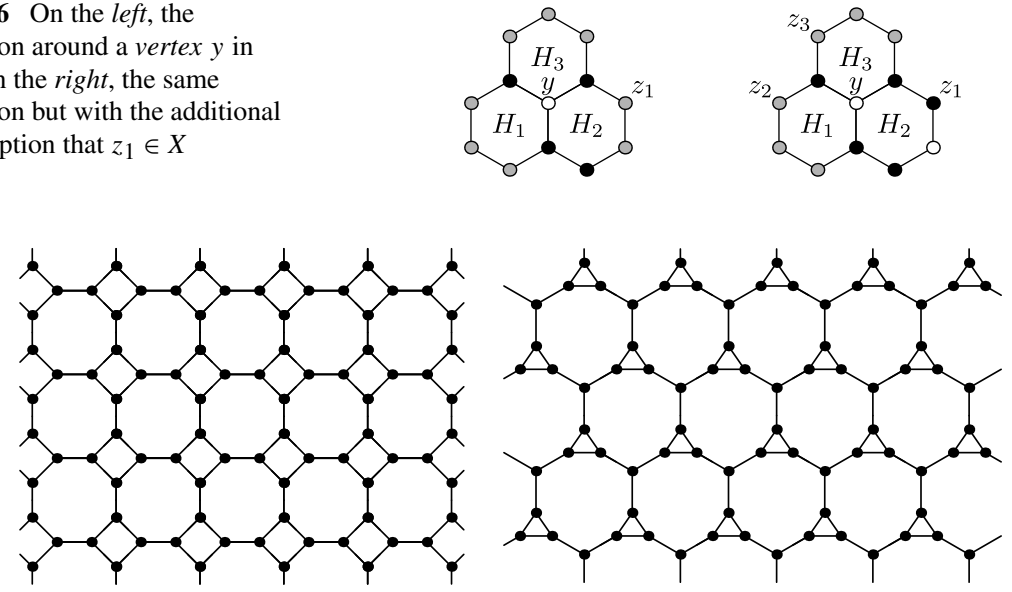

Fig. 17 On the left, the octagon-square grid. On the right, the enneagon-triangle grid

the picture on the right in Fig. 16. Now, if $z_{2} \in X$, then $H_{1}$ belongs to $\mathcal{H}$. If $z_{2}$ does not belong to $X$, then $z_{3} \in X$ and hence $H_{3} \in \mathcal{H}$. This yields the desired claim.

To summarize, since each element in $Y_{3}$ belongs to at least one hexagon in $\mathcal{H}$ and since each hexagon in $\mathcal{H}$ contains exactly one element from $Y_{3}$, we have that $\left|Y_{3}\right| \leq|\mathcal{H}|$. Moreover, since each element in $Y \backslash Y_{3}$ belongs to at most two hexagons in $\mathcal{H}$ and since each hexagon in $\mathcal{H}$ contains exactly two elements from $Y \backslash Y_{3}$, we also have that $2|\mathcal{H}| \leq 2\left|Y \backslash Y_{3}\right|$. As a consequence, $\left|Y_{3}\right| \leq\left|Y \backslash Y_{3}\right|$, which concludes the proof.

Combining Lemmas 7.1 and 7.2, we obtain the following result.

Theorem 7.3 We have that $Q(\mathrm{Hex}, K)=\left[\frac{1}{4}, \frac{5}{18}\right] \cap \mathbb{Q}$.

\section{Some Other 2-Grids}

Fendley and Schoutens [5] computed the (co-)homology of the independence complex of octagon-square and enneagon-triangle grids (see Fig. 17); in each case, all homology is concentrated in one single degree.

Our object is to consider a generalization of the octagon-square grid. Fix $d \geq 1$ and define a $d$-grid $G$ in the following manner; see the picture on the left in Fig. 18 for an illustration. For each integer vector $x \in \mathbb{Z}^{d}$, we have four vertices $w_{x}, n_{x}, s_{x}, e_{x}$ in $G$, and each of $w_{x}$ and $e_{x}$ is adjacent to both $n_{x}$ and $s_{x}$. In addition, for each $x \in \mathbb{Z}^{d}$, $s_{x}$ is adjacent to $n_{x+g_{1}}$, where $g_{1}=(1,0, \ldots, 0)$. Equivalently, for each $x \in \mathbb{Z}^{d}, n_{x}$ is adjacent to $s_{x-g_{1}}$. There might be additional edges in $G$, but there are no other edges containing $n_{x}$ or $s_{x}$ for any $x$. We assume that $G$ is invariant under any transformation $x \mapsto x+y$ (which maps $n_{x}$ to $n_{x+y}$, and so on).

Theorem 8.1 With $G$ defined as above, $Q(G, K)=\left\{\frac{1}{4}\right\}$. In particular, this is true for the octagon-square 2-grid. 

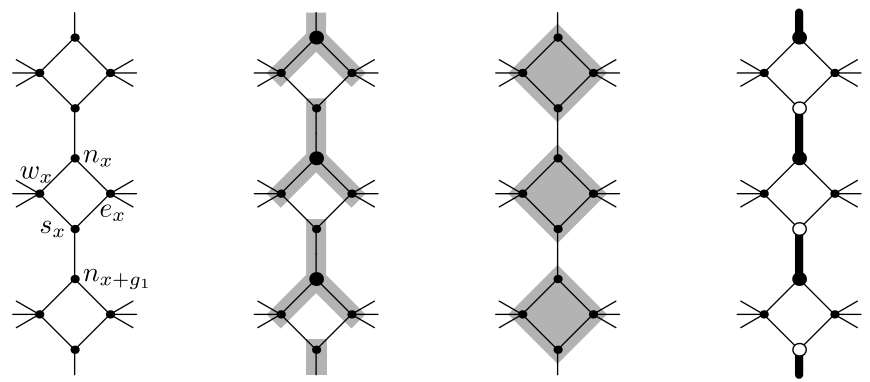

Fig. 18 From left to right, the local shape of the graph $G$ in Theorem 8.1, a partition of $G$ into stars, a partition of $G$ into sets $C_{i}$ satisfying Proposition 3.4, and a matching satisfying Proposition 3.1

Proof We illustrate the constructions of the proof in Fig. 18. Let $I$ be a full-dimension subgroup of $\mathbb{Z}^{d}$. Define $X:=\left\{n_{x}: x \in I\right\}$. It is clear that $X$ satisfies Proposition 3.2, because no $n_{x}$ is adjacent to any $n_{y}$. As a consequence, $G / I$ has no cross-cycles of size below $\left|\mathbb{Z}^{d} / I\right|=\frac{1}{4} \cdot|G / I|$.

Next, define $C_{x}:=\left\{e_{x}, n_{x}, w_{x}, s_{x}\right\}$. It is clear that $\left\{C_{x}: x \in I\right\}$ satisfies Proposition 3.4 with $k_{x}=2$, which immediately yields that $G / I$ has no cross-cycles of size above $\frac{1}{4} \cdot|G / I|$.

Finally, one readily verifies that $\left\{\left(s_{x}, n_{x+g_{1}}\right): x \in I\right\}$ satisfies Proposition 3.1, which yields that $G / I$ contains cross-cycles of size $\frac{1}{4} \cdot|G / I|$.

Using discrete Morse theory [7], it is not hard to prove that $\Sigma(G / I)$ is homotopy equivalent to a wedge of spheres of dimension $\frac{1}{4}|G / I|-1$. For the octagon-square 2 -grid OctSq, Fendley and Schoutens [5] proved that $\Sigma(\mathrm{OctSq} /(m \mathbb{Z} \times n \mathbb{Z}))$ is homotopy equivalent to a wedge of $2^{m}+2^{n}-1$ spheres of dimension $m n-1$.

For the enneagon-triangle grid EnnTri, Fendley and Schoutens demonstrated that EnnTri/I is homotopy equivalent to a wedge of $\alpha_{I}$ spheres of dimension $\frac{1}{4} \mid$ EnnTri $/ I \mid-1$, where $\alpha_{I}$ equals the number of perfect matchings on the hexagonal grid obtained by identifying each triangle in EnnTri/I with a vertex. Their construction is easily seen to imply the following result.

Theorem 8.2 We have that $Q($ EnnTri, $K)=\left\{\frac{1}{4}\right\}$.

\section{Concluding Remarks}

We have computed $Q(G, K)$ for a small selection of 2-grids $(G, K)$. In each case, the outcome was a full interval of rational numbers. As we observed in Sect. 4, $Q(G, K)$ is not always such an interval. Nevertheless, the possibility remains that there exists a reasonably general family of grids, including our examples, with this property.

An important problem for any given grid $G$ is whether cross-cycles tell us "essentially" everything about possible degrees with nonzero homology. More precisely, suppose that $r$ is a rational number such that $r \notin Q(G, K)$. We say that the group $I$ is sparse if the smallest nonzero element in $I$ is large in terms of Euclidean norm. 
- Is it true that $\Sigma(G / I)$ has no homology in degree $r \cdot|G / I|-1$ if $I$ is sparse enough?

- Even better, is there an $\epsilon>0$ such that $\Sigma(G / I)$ has no homology in degree $s \cdot|G / I|-1$ whenever $|s-r|<\epsilon$ if $I$ is sparse enough?

Conversely, for $r \in Q(G, K)$, one may ask whether for each $\epsilon>0$, we have that $\Sigma(G / I)$ has homology in degree $s \cdot|G / I|-1$ for some $s$ such that $|s-r|<\epsilon$ whenever $I$ is sparse enough. This question is closely related to the question as to whether the behavior of $\Sigma(G / I)$ is "fragile" in the sense that a small modification of $I$ may result in a substantially different homology.

We are particularly interested in finding the answers to these questions for the square, triangular, and hexagonal grids.

Acknowledgements This work was carried out at the Technische Universität in Berlin. I thank Günter Ziegler for his kind hospitality and for sponsoring the research via his "Förderpreis für deutsche Wissenschaftler im Gottfried Wilhelm Leibniz-Programm der Deutschen Forschungsgemeinschaft." Furthermore, I thank an anonymous referee for several useful comments on an earlier manuscript. Finally, I thank Alexander Engström, Liza Huijse, and Kareljan Schoutens for inspiring discussions on problems related to this research.

\section{References}

1. Bousquet-Mélou, M., Linusson, S., Nevo, E.: On the independence complex of square grids. J. Algebr. Comb. 27(4), 423-450 (2008)

2. Ehrenborg, R., Hetyei, G.: The topology of the independence complex. Eur. J. Comb. 27, 906-923 (2006)

3. Engström, A.: Complexes of directed trees and independence complexes. Discrete Math. 309(10), 3299-3309 (2009)

4. Engström, A.: Upper bounds on the Witten index for supersymmetric lattice models by discrete Morse theory. Eur. J. Comb. 30(2), 429-438 (2009)

5. Fendley, P., Schoutens, K.: Exact results for strongly-correlated fermions in 2+1 dimensions. Phys. Rev. Lett. 95, 046403 (2005)

6. Fendley, P., Schoutens, K., van Eerten, H.: Hard squares with negative activity. J. Phys. A: Math. Gen. 38(2), 315-322 (2005)

7. Forman, R.: Morse theory for cell complexes. Adv. Math. 134, 90-145 (1998)

8. Huijse, L., Schoutens, K.: Superfrustration of charge degrees of freedom. In: Proceedings of the XXIII IUPAP International Conference on Statistical Physics, Genova, Italy (2007). arXiv:0709.4120v1

9. Huijse, L., Schoutens, K.: Supersymmetry, lattice fermions, independence complexes and cohomology theory. Preprint (2009)

10. Huijse, L., Halverson, J., Fendley, P., Schoutens, K.: Charge frustration and quantum criticality for strongly correlated fermions. Phys. Rev. Lett. 101, 146406 (2008)

11. Jonsson, J.: Hard squares with negative activity and rhombus tilings of the plane. Electron. J. Comb. 13(1), \#R67 (2006)

12. Kozlov, D.: Complexes of directed trees. J. Comb. Theory, Ser. A 88(1), 112-122 (1999)

13. Munkres, J.R.: Elements of Algebraic Topology. Perseus Books (1984)

14. Shareshian, J., Wachs, M.L.: Torsion in the matching complex and chessboard complex. Adv. Math. 212(2), 525-570 (2007)

15. van Eerten, H.: Extensive ground state entropy in supersymmetric lattice models. J. Math. Phys. 46, $123302(2005)$

16. Živaljevič, J.: WI-posets, graph complexes and $\mathbb{Z}_{2}$-equivalences. J. Comb. Theory Ser. A 111, 204$223(2005)$ 